\title{
Gobierno Corporativo y su Revelación a través del Reporte Integrado: Caso MASISA
}

\section{Corporate Governance and its Disclosure Through the Integrated Reporting: MASISA Case}

\author{
José Navarrete Oyarce ${ }^{1}$, José Luis Gallizo Larraz ${ }^{2}$
}

\begin{abstract}
RESUMEN
Este artículo tiene por objetivo analizar las revelaciones sobre aspectos de Gobiernos Corporativos a través del Reporte Integrado, en base de los requerimientos del Marco Internacional del Reporte Integrado. El Reporte Integrado se ha convertido en una fuerte tendencia mundial dentro del ámbito del Reporting.

Para tales efectos, se realizó un estudio de caso, en el informe emitido por la empresa MASISA, dado que esta organización fue la primera empresa chilena en emitir el reporte y una de las primeras a nivel mundial. Se realizó un análisis cualitativo, análisis de contenido, para el Reporte emitido el año 2018.

El análisis demostró un mediano cumplimiento de los requisitos de revelación, dado que se evidenció información de cinco de ocho aspectos revisados. También se determinó la existencia de interconexión entre las revelaciones, en línea con investigaciones previas.

Llama la atención que existan tres aspectos sobre los que no se hace mención alguna. Los resultados también sugieren la utilización del Reporte como mecanismo para mostrar solo aspectos positivos de la organización. Esto abre variadas opciones de investigaciones futuras, tales como, ampliar a otras empresas o revisar el impacto interno de la emisión del Reporte.
\end{abstract}

Palabras claves: gobierno corporativo, reporte integrado, reporte no financiero, revelaciones.

Recepción: 14/05/2019, Aprobación: 20/08/2019

1 Escuela de Ingeniería en Administración de Empresas, Facultad de Economía y Negocios, Universidad Andres Bello, Sede Concepción, Chile. Correo: jose.navarrete@unab.cl

2 Departamento de Administración de Empresas, Facultad de Derecho, Economía y Turismo, Universidad de Lleida, Lleida, España. Correo: gallizo@aegern.udl.cat 


\begin{abstract}
The purpose of this article is to analyse the disclosure on aspects of Corporate Governance through the Integrated Reporting, based on the requirements of the International Framework for Integrated Reporting. The Integrated Reporting has become a strong global trend in the field of Reporting.

To this end, a case study was carried out in the Report issued by the company MASISA, given that this organization was the first chilean company to issue the report and one of the first worldwide. A qualitative analysis, content analysis, was carried out for the Report issued in 2018.

The analysis showed a medium compliance with disclosure requirements, given that there was evidence of five out of eight aspects of information. The existence of interconnection between the disclosures was also determined, in line with previous research.

It is noteworthy that there are three aspects that are not mentioned.

The results also suggest the use of the Report as a mechanism to show only positive aspects of the organization. This opens up a variety of options for future research, such as expanding to other companies or reviewing the internal impact of the issuance of the Report.
\end{abstract}

Keywords: corporate governance, disclosures, integrated reporting, non-financial report.

\title{
INTRODUCCIÓN
}

Las actuales tendencias dejan de lado el secretismo empresarial, siendo la transparencia el paradigma vigente. De esta manera, los stakeholder demandan cada vez más información estratégica, con presencia de aspectos financieros y no financieros (Stewart, 2015; Rupley, Brown, \& Marshall, 2017; Larrán, 2004; Malgioglio \& Fernández, 2012). Este fenómeno tiene variados sustentos conceptuales, tales como la teoría de la legitimidad (Ashforth \& Gibbs, 1990), teoría institucional (Farooq \& Maroun, 2018), teoría de agencia (Villiers \& Hsiao, 2018) y teoría de los stakeholders (Fernández \& Sanjuan, 2012).

En este escenario y dada la abundancia de información y múltiples reportes, se hace difícil establecer una imagen global de la compañía (Jensen \& Berg, 2012; Rivera, Zorio, \& García, 2017).

En función de esto, el Reporte Integrado tiene por objetivo mostrar en tan solo un documento la potencialidad de la empresa para crear valor en el corto y largo plazo, a través de una visión holística de la entidad (Lizcano, Flores, \& Rejón, 2015; IIRC, 2013; Fernández \& Ramón, 2013; Rivera, Zorio, \& García, 2016), y se ha transformado en una innovadora herramienta para informar aspectos esenciales y útiles para las partes interesadas (Hauque \& Rabasedas, 2014). A pesar de que la mayor parte de las empresas que emiten el reporte son europeas (Lopes \& Coelho, 2018), esta tendencia también llegó a Chile, donde ya existe un reducido número de empresas que, voluntariamente han decidido emitir este reporte.

En ese contexto, el análisis de la adopción temprana del Reporte Integrado en Chile se presenta como un problema de investigación relevante. Dado lo anterior, este trabajo tiene como objeti- 
vo analizar las revelaciones asociadas a Gobiernos Corporativos a través del Reporte Integrado, mediante un estudio de caso de la empresa MASISA, la primera en adoptar el formato en Chile y una de las primeras a nivel mundial.

\section{Revisión Bibliográfica}

El Reporte Integrado se define como una comunicación concisa acerca de cómo la estrategia de una organización, su gobierno corporativo, desempeño y perspectivas, en el contexto del entorno, la conducen a crear valor en el corto, mediano y largo plazo (IIRC, 2013).

Esta iniciativa nace de la mano del Consejo Internacional de Reporte Integrado (IIRC por sus siglas en inglés), una coalición global de reguladores, inversores, empresas, emisores de normas, profesionales contables y organizaciones no gubernamentales. En conjunto, esta coalición comparte la visión de que la comunicación acerca de la creación de valor debería ser el próximo paso en la evolución del reporting corporativo (IIRC, 2019)

Los Reportes Integrados combinan los aspectos más relevantes de la información corporativa y, por ende, proporcionan información sobre la estrategia y perspectivas empresariales, dentro de un contexto económico, social y medioambiental (Rivera, Zorio, \& García, 2016).

A diferencia de los Reportes de Sustentabilidad, emitidos en forma aislada, el Reporte Integrado cohesiona aspectos financieros, económicos, de gobierno corporativo y sociales, brindando información sobre las perspectivas futuras con un análisis estratégico, conectando el pasado con el futuro (Rupley, Brown, \& Marshall, 2017; Macias \& Farfan-Lieviano, 2017), haciendo de la empresa una organización más confiable y transparente para los inversores (Hoque, 2017).

El Reporte Integrado debe mostrar el camino para que la empresa logre incorporar su visión y valores, conceptos que deben emerger desde la alta administración, con monitoreo constante del gobierno corporativo, a través del uso de los distintos capitales (Abeysekera, 2013).

Entre los aspectos positivos evidenciados con la emisión del reporte, se tiene una disminución de errores por parte de los analistas financieros (Zhou, Simnett, \& Kelly, 2017), una relación positiva entre emisión del Reporte Integrado y valorización bursátil, efecto más visible en grandes compañías con intangibles (Lee \& Yeo, 2016), reducción de costos de capital (Ortiz-Martinez \& Marín-Hernández, 2016), relación positiva entre el Reporte Integrado y el return on invested capital, ROIC (Churet \& Eccles, 2014), entre otros.

No obstante, el Reporte Integrado es concebido como una herramienta de contabilidad asociada al concepto de sustentabilidad, a juicio de Flower (2015), este objetivo no se cumple, puesto que se establece la idea de "valor para el inversionista" y no "valor para la sociedad". En ese mismo contexto, Flower (2015) cree que el IIRC finalmente ha tenido un impacto menor en las prácticas de reporting empresarial. En la misma línea Reuter \& Messner (2015) establecen que, en la confección del Marco Conceptual del Reporte, participaron fundamentalmente grandes compañías, en desmedro de las pequeñas y medianas, con un fuerte lobby de entidades preparadoras de reportes versus usuarios de información. En concordancia con esto Gibassier, M., \& Arjalies (2018), indican que muchas empresas desafiaron la visión de Reporte Integrado sugerida por el IIRC y finalmente optaron por implementar su propia versión de un informe. A su vez, Dumay, 
Bernardi, Guthrie, \& La Torre (2017) señalan que la flexibilidad, así como los criterios de medición, pueden generar que la emisión del reporte sea solo con fines de cumplimiento, sin lograr el resto de los beneficios del informe. Debido a las diferentes formas en que el Reporte es entendido, existen numerosos desafíos teóricos y empíricos para los académicos (Dumay, Bernardi, Guthrie, \& La Torre, 2017).

Por otro lado, la complejidad del lenguaje utilizado en los Reportes Integrados, dificultan su entendimiento y, en consecuencia, afecta a los grupos de interés, en su búsqueda de información (Du Toit, 2017). De la misma forma, otro aspecto discutible del Reporte Integrado está en el hecho que muchos actores lo perciban como un instrumento para mostrar las bondades de una organización, lo que le resta credibilidad (Rejón, Flores, \& Lizcano, 2013). En ese sentido, aunque el IIRC no aspira a ser un elemento gravitante en el mundo del assurance, si reconoce los beneficios de establecer mecanismos para construir confianza (IIRC, 2014).

Dentro de los contenidos relevantes que el Reporte Integrado debe revelar, está lo relativo a Gobierno Corporativo, instancia que tiene como objetivo generar los medios necesarios para prevenir y corregir posibles conflictos de interés, entre las partes interesadas, en el marco de la teoría de agencia de (Jensen \& Meckling, 1976).

Con respecto a Gobiernos Corporativos y su interacción con el mercado chileno, existen una serie de estudios previos en los que se realizan análisis de diversas naturalezas (Lefort, F., \& Urzúa, F., 2008; Lefort, F., \& Walker, E., 2007; Jara, M., López-Iturriaga, F., San-Martín, P., \& Saona, $P, 2019)$. Ahora bien, respecto a gobierno corporativo y su revelación en el mercado chileno, trabajos anteriores determinan un bajo grado de adopción de las prácticas de gobierno corporativo entre el grupo de las empresas más grandes de Chile, lo que hace dudar acerca del compromiso con la transparencia por parte de ellas, al considerar poco conveniente revelar información a los usuarios (Moraga \& Ropero, 2018). Adicionalmente, se evidencia una baja adopción respecto a prácticas de gobiernos corporativo en Chile, así como un alto grado de heterogeneidad en los sectores a los que pertenecen estas empresas (Moraga \& Rossi, 2019).

\section{METODOLOGIA, MATERIALES Y MÉTODOS}

\section{Metodología de la Investigación}

Sobre la base que el Reporte Integrado es un tema relativamente nuevo en el mundo empresarial, una mirada cualitativa es la más apropiada, dada la necesidad de explorar y describir un fenómeno y desarrollar, sobre ello, una teoría (Creswell, 2013). De la misma forma, a través del estudio de casos, es factible responder preguntas tales “cómo" y "por qué", los que están orientados a campos incipientes de investigación (Eisenhardt \& Graebner, 2007).

Dado lo anterior, este trabajo utiliza un enfoque cualitativo, analizando el caso de la empresa MASISA, la primera empresa chilena y una de las primeras a nivel mundial, en emitir Reportes Integrados. Con estas referencias, el caso cumple con los criterios de singularidad, complejidad, disponibilidad y potencial de aprendizaje, indicados por la literatura para validar un caso (Durán, 2012). Para tales efectos, se debe mencionar que según las indicaciones del IIRC, un Reporte Integrado debe cumplir una serie de directrices, así como revelar cierto contenido mínimo (IIRC, 
2013), tal y como se aprecia en el Cuadro $N^{\circ} 1$ Si bien existe este documento base, es necesario precisar que no se cuenta con requisitos fijos para el contenido de un Reporte Integrado, por lo que una parte de él quedará necesariamente a criterio de la empresa que lo está confeccionado.

Se realizó un análisis de contenido en base a los requerimientos de "contenido" (IIRC, 2013), con metodología propia, tomando en cuenta los elementos que establece el IIRC y que deberían estar presentes en los reportes integrados, tomando como base trabajos anteriores, tales como Stent \& Dowler (2015), Sofian \& Dumitru (2017), Rivera, Zorio, \& García (2017).

\section{Cuadro 1}

Requerimientos del Reporte Integrado

\begin{tabular}{l|l}
\hline Relativos a las Directrices & Relativos al Contenido \\
\hline 1.- Enfoque estratégico y orientación futura. & 1.- Descripción de la entidad y su entorno. \\
2.- Conectividad de la información. & 2.- Gobierno Corporativo. \\
3.- Relación con los grupos de interés. & 3.- Modelo de negocio. \\
4.- Materialidad. & 4.- Riesgos y oportunidades. \\
5.- Concisión. & 5.- Estrategia y asignación de recursos. \\
6.- Fiabilidad e integridad. & 6.- Desempeño. \\
7.- Comparabilidad y consistencia & 7.- Perspectivas futuras. \\
& 8.- Bases de preparación y presentación. \\
\hline
\end{tabular}

Nota: Elaboración propia en base a (IIRC, 2013).

En forma específica, se analizó el concepto de "Gobierno Corporativo", dentro de los contenidos que debe tener un Reporte Integrado. De esta forma se generaron ocho códigos de análisis, vale decir, frases representativas de un elemento o síntesis cuyo objetivo es capturar la esencia o atributo. Estos códigos son de naturaleza deductiva, toda vez que fueron establecidos en forma previa, de la revisión analítica del Marco Internacional del IIRC (IIRC, 2013). Para un mejor entendimiento del análisis, el detalle de códigos definidos puede apreciarse en Anexo $\mathrm{N}^{\circ} 1$. El análisis fue realizado al Reporte Integrado emitido por la empresa MASISA el año 2018, disponible en su página web.

\section{Acerca de MASISA}

MASISA es una empresa dedicada a la fabricación y comercialización de tableros de madera (MDF y MDP / PB) para muebles y arquitectura de interiores, en el ámbito latinoamericano (Masisa, 2019). Posee 10 complejos industriales en Chile, Argentina, Brasil, Venezuela y México, con una capacidad instalada de producción de tableros de $3.427 \mathrm{mil} \mathrm{m3}$, siendo la mayor compañía en Latinoamérica (ex Brasil). Tiene 224 mil hectáreas de plantaciones de pino y eucaliptus en Chile, Argentina, Brasil y Venezuela, las que son manejadas bajo altos estándares medio ambientales (FSC). Su unidad de retail cuenta con un canal de distribución estratégico, Placacentro, con 345 tiendas, de las cuales, 50 son propias (Bolsa de Santiago, 2019). 
MASISA lleva a cabo sus negocios, a través de una estrategia de Triple Resultado, el que contempla, de forma integral y simultánea, obtener los más altos índices de calidad en su desempeño, tanto en el ámbito financiero, social y medioambiental (Masisa, 2019). Sobre la base de esta filosofía de trabajo, MASISA emite Reporte Integrado desde el año 2011, siendo la primera empresa chilena en hacerlo y una de las primeras a nivel mundial, en adoptar el formato.

\section{RESULTADOS}

Es posible determinar que existe un mediano cumplimiento de los requisitos de revelación de aspectos de Gobiernos Corporativos en el documento analizado, toda vez que se logró rescatar información respecto a 5 de los 8 conceptos establecidos. En consecuencia, llama la atención que no haya revelaciones asociadas a los códigos B3, Fuerzas de mercado, competencia y clientes; B7, Promoción de la innovación y B8, Incentivos ligados a uso de capitales. También se debe mencionar que existen mayoritariamente revelaciones escritas (dentro del cuerpo del documento) por sobre esquemas o imágenes. En Tabla $N^{\circ} 2$ se presenta relación de códigos y sus respectivas frecuencias de aparición en el documento:

\section{Tabla 1}

\section{Resultados Análisis de Contenido}

\begin{tabular}{|l|c|}
\hline \multicolumn{1}{|c|}{ Códigos } & Frecuencia \\
\hline B1: Estructura de liderazgo, competencias y diversidad, requisitos regulatorios. & 6 \\
\hline $\begin{array}{l}\text { B2: Toma de decisiones estratégicas, monitoreo, actitud frente al riesgo, evalua- } \\
\text { ción de integridad y ética. }\end{array}$ & 9 \\
\hline $\begin{array}{l}\text { B3: Fuerzas del mercado, fortalezas y debilidades de la competencia y demanda } \\
\text { de clientes. }\end{array}$ & 0 \\
\hline $\begin{array}{l}\text { B4: Acciones para influir y supervisar la dirección estratégica y enfoque de ges- } \\
\text { tión de riesgo. }\end{array}$ & 7 \\
\hline $\begin{array}{l}\text { B5: Cultura, ética y valores y como se reflejan en el uso de los capitales, relación } \\
\text { con grupos de interés. }\end{array}$ & 3 \\
\hline B6: Prácticas de gobierno corporativo que superen los requerimientos legales. & 5 \\
\hline $\begin{array}{l}\text { B7: Responsabilidad del gobierno corporativo para promover y facilitar la inno- } \\
\text { vación. }\end{array}$ & 0 \\
\hline $\begin{array}{l}\text { B8: Remuneración e incentivos ligados a la creación de valor y su relación con el } \\
\text { uso de los capitales. }\end{array}$ & 0 \\
\hline
\end{tabular}

Fuente: Elaboración propia en base a Reporte Integrado Masisa 2018.

En forma complementaria, al analizar los resultados en formato de red, puede apreciarse que el código B2, Toma de decisiones estratégicas, está relacionado con el código B4, Acciones para influir en la estrategia y B6, Prácticas por sobre los estándares. De la misma manera B1, Estructura de gobierno corporativo está relacionado con B2, Toma de decisiones estratégicas. Esto quiere decir que, en un mismo párrafo, esquema o imagen, se satisface la revelación de más de un con- 
cepto, en línea con la directriz de conectividad de la información, establecida por el IIRC (IIRC, 2013) y evidenciado en trabajos anteriores (Rivera-Arrubla \& Zorio-Grima, 2016). Finalmente, B5, Cultura, ética y valores no tiene ninguna relación con los otros. Detalle de las interacciones, se puede revisar en Figura $\mathrm{N}^{\circ} 1$.

Es interesante ver que no se hace ninguna alusión directa a la interacción de los órganos de gobierno corporativo con los capitales indicados en por el IIRC (IIRC, 2013), y que son pieza fundamental del concepto "Integrado" del reporte.

\section{Figura 1}

Red Análisis de Contenido

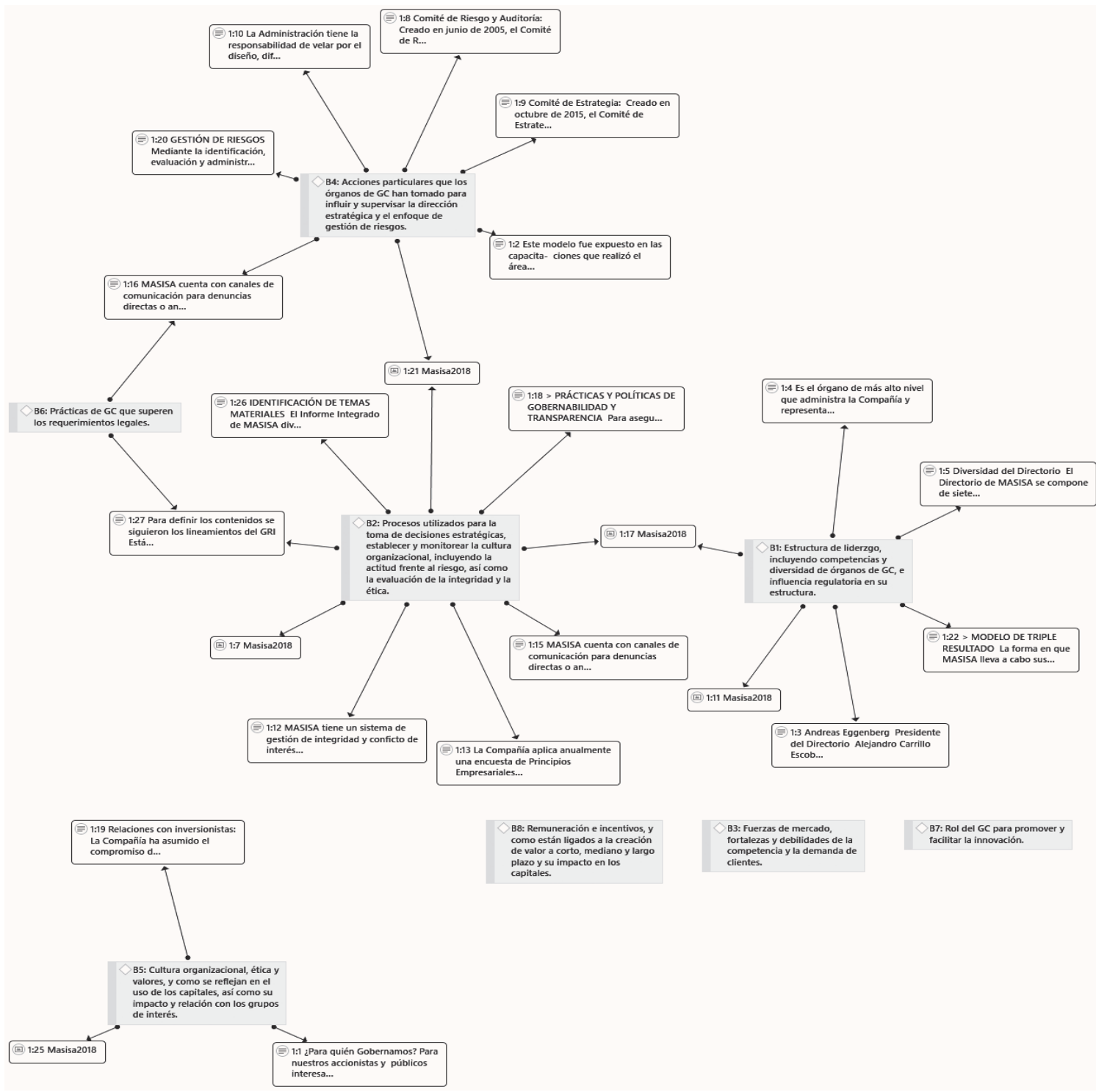

Fuente: Elaboración propia en base a Reporte Integrado MASISA 2018 y generada por software ATLAS.ti. 


\section{DISCUSIÓN Y CONCLUSIONES}

Se puede apreciar un mediano nivel de cumplimiento en torno a las revelaciones de gobiernos corporativos, consistente con trabajos anteriores (Rivera, Zorio, \& García, 2017), siendo el concepto más revelado el que tiene relación con los procesos utilizados para la toma de decisiones estratégicas, con énfasis en gestión de riesgos y aspectos éticos.

Es importante destacar que, dentro del documento, se hacen varias alusiones a los procedimientos éticos y de buenas prácticas que la empresa realiza, tales como el proceso de autoevaluación del directorio o el canal para denuncias sobre la observación de prácticas empresariales, por ejemplo, prácticas que superarían el estándar de la industria. También se percibe una alta importancia a las revelaciones asociadas a la gestión de riesgo. Esto podría asociarse a la utilización de este mecanismo como un vehículo para potenciar los aspectos positivos de la organización (Rejón, Flores, \& Lizcano, 2013). Se rescata el uso de textos sobre imágenes o esquemas, como vehículos de comunicación, situación revisada en trabajos anteriores (Rivera-Arrubla \& Zorio-Grima, 2016).

Llama la atención, que no existe alusión a como los órganos de gobiernos corporativo promueven y/o facilitan la innovación, así como tampoco existen claridad en como los incentivos están ligados a la creación de valor para la compañía ni cómo interactúan con los diversos capitales. Si bien se hace alusión a la remuneración del directorio, no se hace la relación de las metas estratégicas asociadas ni los incentivos, probablemente por considerar esta información como estratégica, consistente con estudios previos (Dumay, Bernardi, Guthrie, \& La Torre, 2017; Gibassier, M., \& Arjalies, 2018). Finalmente, tampoco se hace referencia a las fuerzas del mercado, competencia ni demanda, desde la óptica de su relación con el Gobierno Corporativo.

A pesar de las limitantes de esta investigación, se puede determinar que varios de los escenarios previstos en la literatura, se pueden apreciar en este caso, tal y como la implementación de versiones propias (Gibassier, M., \& Arjalies, 2018), o la emisión con fines de cumplimiento (Dumay, Bernardi, Guthrie, \& La Torre, 2017). De esta forma, los pasos próximos estarán dados por realizar este mismo estudio en otras compañías nacionales, así como contrastar estas revelaciones desde el punto de vista interno de la organización.

\section{REFERENCIAS BIBLIOGRAFICAS}

Abeysekera, I. (2013). A template for integrated reporting. Journal of Intellectual Capital, 227-245. Ashforth, B., \& Gibbs, B. (1990). The double-edge of organizational legimitation. Organization Science, 177-194.

Bolsa de Santiago. (15 de 05 de 2019). Obtenido de http: / / www.bolsadesantiago.com/InfoInstrumento / Paginas / ResenaCompania.aspx?Nemo=MASISA

Churet, C., \& Eccles, R. (2014). Integrated Reporting, quality of management and financial perfomance. Journal of Applied Corporate Finance, 56-64.

Creswell, J. (2013). Research design: Qualitative, Quantitative, and Mixes Methods Approaches. California: SAGE Publications Inc.

Du Toit, E. (2017). The readability of integrated reporting. Meditari Accountancy Research, 629653. 
Dumay, J., Bernardi, C., Guthrie, J., \& La Torre, M. (2017). Barriers to implementing the international integrated reporting framework. Meditari Accountancy Research, 461-480.

Durán, M. (2012). El estudio de caso en la investigación cualitativa. Revista Nacional de Administración, 121-134.

Eisenhardt, K., \& Graebner, M. (2007). Theory building from cases: opportunities and challenges. The Academia of Management Journal, 25-32.

Farooq, M., \& Maroun, W. (2018). Sustanitability accounting and integrated reporting - Chapter 4. Abingdon, UK: Routledge.

Fernández, E., \& Ramón, R. (2013). Revelación de la información financiera y no financiera: Informes Integrados. Revista Contable, 78-96.

Fernández, J. L., \& Sanjuan, A. (2012). La Teoría del Stakeholder o de los Grupos de Interés, pieza clave de la RSE, del éxito empresarial y de la sostenibilidad. International Journal of Communication Research, 130-143.

Flower, J. (2015). The integrated reporting council: a story of failure. Critical Perspectives on Accounting, 1-17.

Gibassier, D., M., R., \& Arjalies, D. (2018). “Integrated reporting is like a God: no one has meet Him, but everybody talks about Him": The power of myths in the adoption of management innovations. Accounting, Auditing \& Accountability Journal, 1349-1380.

Hauque, S., \& Rabasedas, M. (2014). Reportes Integrados: la integración de información bajo la visión del International Integrated Reporting Council (IIRC). Capic Review, 67-92.

Hoque, M. (2017). Why companies should adopt Integrated Reporting? International Journal of Economics and Finance, 241-248.

IIRC. (2013). El Marco Internacional $<\mathrm{IR}>$. Londres.

IIRC. (2014). Assurance on $<\mathrm{IR}>$ : an introduction to the discussion. Londres.

IIRC. (15 de 05 de 2019). Obtenido de https: / / integratedreporting.org/

Jara, M., López-Iturriaga, F., San-Martín, P., \& Saona, P. (2019). Corporate governance in Latin American firms: Contestability of control and firm value. BRQ Business Research Quarterly, 22(4), 257-274.

Jensen, J., \& Berg, N. (2012). Determinants of traditional sustaintability reporting versus integrated reporting, an institutional approach. Business Strategy and the Enviroment, 299-316.

Jensen, M., \& Meckling, W. (1976). Theory of the firm: Managenial behavior, agency cost, and ownership structure. Journal of Financial Economics, 305-360.

Larrán, M. G.-M. (2004). La relevancia de la información . Revista Valenciana de Economía y Hacienda, 127-145.

Lee, K.-W., \& Yeo, G. H.-H. (2016). The association between integrated reporting and firm valuation. Review of Quantitative Finance and Accounting, 1221-1250.

Lefort, F., \& Urzúa, F. (2008). Board independence, firm performance and ownership concentration: Evidence from Chile. Journal of Business Research, 61(6), 615-622.

Lefort, F., \& Walker, E. (2007). Do markets penalize agency conflicts between controlling and minority shareholders? Evidence from Chile. The Developing Economies, 45(3), 283-314.

Lizcano, J., Flores, F., \& Rejón, M. (2015). Nuevos horizontes de regulación sobre información no financiera, norma integrada, norma europea y propuesta de AECA. Revista Contable, 92105.

Lopes, A., \& Coelho, A. (2018). Engaged in integrated reporting? Evidence across multilpe organizations. European Business Review, 398-426. 
Macias, H., \& Farfan-Lieviano, A. (2017). Integrated Reporting as a strategy for firm growth: multiple case study in Colombia. Meditari Accountancy Research, 16-48.

Malgioglio, J., \& Fernández, R. (2012). La importancia de la información no financiera para toma de decisiones. Decimoséptima Jornada de Investigación en Facultad de Cs. Económicas y Estadísticas. Rosario, Argentina.

Masisa. (15 de 05 de 2019). Estrategia de Triple Resultado. Obtenido de www.masisa.com/nosotros/nuestra-empresa/ principios-y-valores

Masisa. (14 de 05 de 2019). Nuestra Empresa. Obtenido de www.masisa.com/nosotros/nuestraempresa

Moraga, H., \& Ropero, E. (2018). Gobierno corporativo y desempeño financiero de las empresas más importantes del mercado bursátil chileno. Revista Venezolana de Gerencia, 19-38.

Moraga, H., \& Rossi, M. (2019). Gobierno corporativo y riesgo de quiebra en las empresas chilenas. Globalización, Competitivas y Gobernabilidad de Georgetown/Universia, 113-125.

Ortiz-Martinez, E., \& Marín-Hernández, S. (2016). Comunicar informació no financiera e IDE por regiones. Globalización, Competitividad y Gobernabilidad, 94-111.

Rejón, M., Flores, F., \& Lizcano, J. (2013). La verificación de la información integrada: el papel de las firmas de auditoria. Revista Contable, 66-75.

Reuter, M., \& Messner, M. (2015). Lobbying on integrated reporting framework. Accounting, Auditing \& Accountability Journal, 365-402.

Rivera, Y., Zorio, A., \& García, M. (2016). El concepto de Informe Integrado como innovación en el Reporting Corporativo. Journal of Innovation \& Knowledge, 144-155.

Rivera, Y., Zorio, Z., \& García, M. (2017). Integrated Reports: disclosure level and explanatory factors. Social Reponsability Journal, 155-176.

Rivera-Arrubla, Y., \& Zorio-Grima, A. (2016). Integrated reporting, connectivity, and social media. Psychology \& Marketing, 1159-1165.

Rupley, K., Brown, D., \& Marshall, S. (2017). Evolution of corporate reporting: from state-alone corporate social responsability to integrated reporting. Research in Accounting Regulation, 1-5.

Sofian, I., \& Dumitru, M. (2017). The compliance of the integrated reporting issued by european financial companies with international integrated reporting framwork. Sustainability, 1159-1165.

Stent, W., \& Dowler, T. (2015). Early assessment of the gap between integrated reporting and current corporate reporting. Meditaria Accountancy Research, 92-117.

Stewart, L. (2015). Growing demand for ESG Information and Standar: Understanding Corporate Oportunities as Well Risk. Journal of Applied Corporate Finance, 58-63.

Villiers, C., \& Hsiao, P. (2018). Sustanitability accounting and integrated reporting - Chapter 5. Abingdon, UK: Routledge.

Zhou, S., Simnett, R., \& Kelly, P. (2017). Does Integrated Reporting matter to the capital market? A Journal of Accounting Finance and Business Studies, 94-132. 


\section{ANEXO N ${ }^{\circ}$ : \\ Revelaciones de la estructura de gobierno corporativo requeridas en un Reporte Integrado}

\section{Gobierno Corporativo.}

¿Cómo funciona la estructura de gobierno corporativo en una organización para apoyar su habilidad de crear valor a corto, medio y largo plazo?

\begin{tabular}{|c|}
\hline $\begin{array}{l}\text { 1.- La estructura de liderazgo en la organización, incluyendo las competencias y la di- } \\
\text { versidad (p.ej. el espectro de formaciones, género, competencias y experiencias) de los } \\
\text { órganos de gobierno corporativo, y si los requisitos regulatorios influyen en el diseño de } \\
\text { la estructura de gobierno corporativo. }\end{array}$ \\
\hline $\begin{array}{l}\text { 2.- Los procesos específicos utilizados para la toma de decisiones estratégicas, y para el } \\
\text { establecimiento y monitorización de la cultura de la organización, incluyendo su actitud } \\
\text { frente al riesgo y los mecanismos para evaluar la integridad y las cuestiones éticas. }\end{array}$ \\
\hline $\begin{array}{l}\text { 3.- Las fuerzas del mercado, tales como las fortalezas y debilidades de los competidores, } \\
\text { y la demanda de los clientes. }\end{array}$ \\
\hline $\begin{array}{l}\text { 4.- Las acciones particulares que los órganos de gobierno corporativo han tomado para } \\
\text { influir y supervisar la dirección estratégica de la organización y su enfoque en la gestión } \\
\text { del riesgo. }\end{array}$ \\
\hline $\begin{array}{l}\text { 5.- Cómo la cultura de la organización, la ética y valores se reflejan en el uso que se da } \\
\text { a los capitales y su impacto, incluyendo sus relaciones con los grupos de interés clave. }\end{array}$ \\
\hline $\begin{array}{l}\text { 6.- Si la organización está implementando prácticas de gobierno corporativo que superan } \\
\text { los requerimientos legales. }\end{array}$ \\
\hline $\begin{array}{l}\text { 7.- La responsabilidad que asumen los órganos de gobierno corporativo para promover } \\
\text { y facilitar la innovación. }\end{array}$ \\
\hline $\begin{array}{l}\text { 8.- Cómo la remuneración e incentivos están ligados a la creación de valor al corto, me- } \\
\text { dio y largo plazo, incluyendo cómo están ligados al uso de y al impacto de los capitales } \\
\text { en la organización. }\end{array}$ \\
\hline
\end{tabular}

